\title{
SSynthesis
}

International Scientific Conference of IT and Business-Related Research

\section{GUSTINA VEROVATNOĆE SIGNALA NA IZLAZU IZ MAKRODIVERZITI SISTEMA SA TRI MIKRODIVERZITI SC PRIJEMNIKA U PRISUSTVU BRZOG K- $\mu$ FEDINGA I GAMA SPOROG FEDINGA}

\author{
PROBABILITY DENSITY FUNCTION OF A SIGNAL LEAVING MACRO-DIVERSITY SYSTEM \\ WITH THREE MICRO- DIVERSITY SC RECEPTORS IN THE PRESENCE OF FAST \\ $\mathrm{K}-\mu$ FADING AND SLOW GAMMA FADING
}

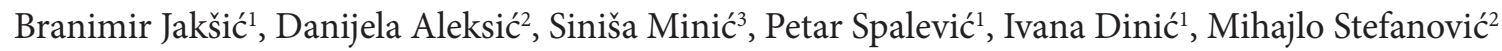 \\ ${ }^{1}$ Fakultet tehničkih nauka, Kneza Miloša, 7, Kosovska Mitrovica, Srbija \\ ${ }^{2}$ Elektronski fakultet, Aleksandra Medvedva, 14, Niš, Srbija \\ ${ }^{3}$ Učiteljski fakultet, Leposavić, Srbija
}

\begin{abstract}
Apstrakt:
$\mathrm{U}$ ovom radu razmatran je diverziti sistem sa tri mikrodiverziti SC prijemnika i jednim makrodiverziti SC prijemnikom. Na ulazima u mikrodiverziti SC prijemnike prisutan je nezavisni k- $\mu$ feding i korelisani spori Gama feding. Za ovaj model izračunati su gustina verovatnoća signala na izlazu iz mikrodiverziti SC prijemnika i makrodiverziti SC prijemnika. Dobijeni rezultati su grafički prikazani kako bi se pokazao uticaj Rajsovog k faktora, dubine osenčenosti kanala c, broj klastera $\mu$ i koeficijenta korelacije $\rho$ na gustinu verovatnoće signala na izlazu iz makrodiverziti sistema.
\end{abstract}

\section{Ključne reči:}

Rajsov k faktor, koeficijent korelacije, makrodiverziti SC prijemnik, mikrodiverziti SC prijemnik.

\section{UVOD}

U bežičnim mobilnim telekomunikacionim sistemima osnovna vrsta smetnji je feding. Mogu nastati razne vrste fedinga zavisno od propagacione okoline i komunikacionog scenarija. Brzi feding nastaje zbog prostiranja signala po više puteva. Naime, interakcija talasa sa objektima koji se nalaze između predajnika i prijemnika (refleksija, difrakcija i rasejanje) prouzrokuje da na ulaz prijemnika stiže veliki broj kopija poslatog signala. Superpozicija kopija izvornog signala koje se razlikuju po kašnjenju, faznom pomeraju i slabljenju dovodi do toga da snaga signala na ulazu prijemnika nije stalna veličina. Sredina kroz koju se talas prostire može da bude linearna i nelinearna. Sredina je nelinearna kada su površine od kojih se vrši odbijanje talasa korelisane tako da polje rasipanja nije homogeno (Stuber, 2003; Panić et al, 2013).

Spori feding nastaje zbog efekta senke. Efekat senke mogu formirati razni objekti između predajnika i prijemnika (Simon et al, 2000). U većini slučajeva spori feding je korelisan. Prome-

\section{Abstract:}

This paper examines the diversity system with three micro-diversity SC receptors and one macro- diversity SC receptor. Independent $\mathrm{k}-\mu$ fading and correlated slow Gamma fading are observed at the entrance into micro- diversity SC receptors. Probability density functions of a signal leaving micro-diversity SC receptor and macro-diversity SC receptor is calculated for the given model. The results obtained are graphically presented to indicate the impact of Rician k-factor, fading figure of channel c, number of clusters $\mu$ and correlation coefficient $\rho$, on the probability density function of a signal leaving micro-diversity system.

\section{Key words:}

Rician k factor, correlation coefficient, macro-diversity SC receptor, macro-diversity SC receptor.

na snage signala zbog uticaja efekta senke su spore u odnosu na promenu anvelope signala zbog brzog fedinga. Zavisno od propagacione okoline, snage komponente $u$ fazi i komponente $\mathrm{u}$ kvadraturi signala na prijemniku mogu da budu iste ili različite. Anvelopa signala je promenljiva zbog brzog fedinga, a snaga anvelope signala je promenljiva zbog sporog fedinga (Panić et al, 2011; Yacoub, 2007). Statističko ponašanje signala u ovakvim sistemima može se opisati različitim raspodelama: Rejlijevom, Rajsovom, Nakagami- $m$, Vejbulovom ili k- $\mu$. $k$ - $\mu$ raspodela može biti upotrebljena da opiše varijaciju anvelope signala $u$ linearnim sredinama gde postoji dominantna komponenta, postoji više klastera u propagacionoj okolini i snage komponente u fazi i kvadraturi su jednake. $k$ - $\mu$ raspodela ima dva parametra. Parametar $k$ je Rajsov faktor i jednak je količniku snage dominantne komponente i snage linearnih komponenti. Parametar $\mu$ je povezan sa brojem klastera $u$ propagacionoj okolini. $k-\mu$ raspodela je generalna raspodela (Proakis, 2001; Shankar, 2008). Rajsova, Nakagami- $m$ i Rejlijeva raspodela mogu se dobiti iz $k-\mu$ raspodele kao specijalni slučajevi (Sekulović et al, 2012). 
Koriste se razne diverziti tehnike da se smanji uticaj brzog fedinga i sporog fedinga na performanse sistema. Kod diverziti tehnika više replika istog informacionog signala se kombinuje. Najčešće se koriste prostorne diverzite tehnike. Prostorne diverziti tehnike su realizovane sa više antena postavljenih na prijemniku. Primenom prostornih diverziti tehnika povećava se pouzdanost sistema i kapacitet kanala bez povećanja snage predajnika i širenja frekventnog opsega. Postoji više prostornih diverziti tehnika kombinovanja koje se mogu upotrebiti da se smanji uticaj fedinga i međukanalne interferencije na performanse sistema. Najčešće korišćene diverziti tehnike su MRC (maximum ratio combining), EGC (equal gain combining) i SC (selection combining) (Stuber, 2003; Proakis, 2001). SC diverziti prijemnik je jednostavan za praktičnu realizaciju zbog toga što se procesiranje vrši samo na jednoj diverziti grani. SC prijemnik izdvaja granu sa najvećim odnosom signala i šuma. Ako je snaga šuma ista u svim granama onda SC prijemnik izdvaja granu sa najjačim signalom.

\section{MODEL SISTEMA}

U ovom radu razmatra se makrodiverziti sistem sa makrodiverziti SC (selection combining) prijemnikom i tri mikrodiverziti SC prijemnika. Na ulazima u mikrodiverziti SC prijemnike prisutan je nezavisni $k$ - $\mu$ feding i spori Gama feding. Spori $f e-$ ding je korelisan. Koeficijent korelacije opada sa rastojanjem između antena.

Mikrodiverziti SC prijemnik smanjuje uticaj brzog fedinga na performanse sistema, a makrodiverziti SC prijemnik smanjuje uticaj sporog fedinga na performanse sistema. Makro sistem koji se razmatra može biti primenjen u jednoj ćeliji, ćelijskog mobilnog radio sistema. Mikrodiverziti prijemnici se postavljaju na baznim stanicama koje opslužuju mobilne korisnike u jednoj ćeliji. Makrodiverziti sistem koristi signale od više baznih stanica postavljenih u jednoj ćeliji ili dve i više ćelija.
Sistem koji se razmtra prikazan je na Slici 1.

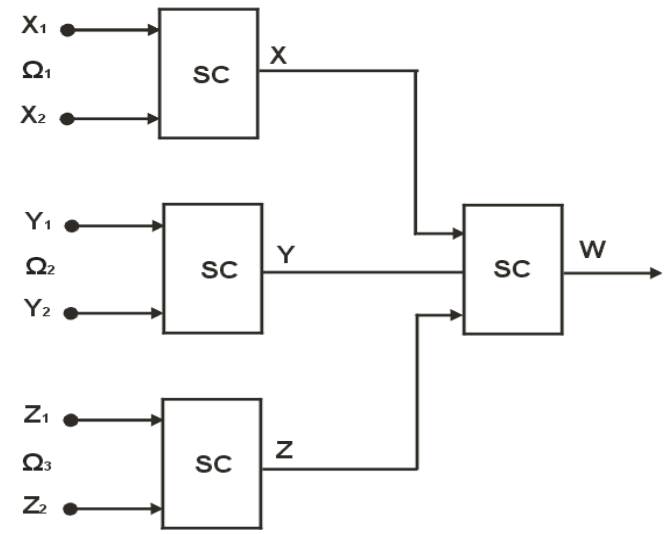

Slika 1. Makrodiverziti sistem sa tri mikrodiverziti MRC kombinera.

Signali na ulazima u prvi SC mikrodiverziti prijemnik su označeni sa $x_{1}$ i $x_{2}$, a na izlazu $x$. Signali na ulazima u drugi SC mikrodiverziti prijemnik su označeni sa $y_{1}$ i $y_{2}$, a na izlazu $y$. Signali na ulazima u treći SC mikrodiverziti prijemnik su označeni sa $z_{1}$ i $z_{2}$, a na izlazu $z$. Signal na izlazu iz makrodiverziti sistema je označen sa $w$. Snage signala na ulazima u mikrodiverziti prijemnike su označene sa $\Omega_{1}, \Omega_{2}$ i $\Omega_{3}$.

Signal na izlazu iz makrodiverziti SC prijemnika $w$ jednak je signalu na izlazu iz onog mikrodiverziti SC prijemnika čija je snaga na ulazu veća od snage signale na ulazu ostalih mikrodiverziti SC prijemnika (Panić et al, 2013).

\section{GUSTINA VEROVATNOĆE SIGNALA}

Gustina verovatnoće $k-\mu$ signala $x_{1}$ i $x_{2}$ data je sa

$$
p_{x_{i}}\left(x_{i}\right)=\frac{2 \mu(k+1)^{\frac{\mu+1}{2}}}{k^{\frac{\mu-1}{2}} e^{\mu k} \Omega_{1}^{\mu+1}} x_{i}^{\mu} e^{-\frac{\mu(k+1) x_{i}^{2}}{\Omega_{1}}} I_{\mu-1}\left(2 \mu \sqrt{\frac{k(k+1)}{\Omega_{1}}} x_{i}\right), i=1,2
$$

Parametar $\mu$ predstavlja broj klastera kroz koji se prostire signal, $k$ Rajsov faktor, $x_{0}$ srednja snaga signala, a $I_{n}(\cdot)$ modifikovana Beselova funkcija prvog reda $n$-te vrste. Nakon razvoja Beselove funkcije u red, izraz za gustinu verovatnoće $x$ postaje (Gradshteyn, 2000):

$$
p_{x_{i}}\left(x_{i}\right)=\frac{2 \mu(k+1)^{\frac{\mu+1}{2}}}{k^{\frac{\mu-1}{2}} e^{\mu k} \Omega_{1}^{\mu+1}} x_{i}^{\mu} e^{-\frac{\mu(k+1) x_{i}^{2}}{\Omega_{1}}} \sum_{i_{1}=0}^{\infty}\left(\mu \sqrt{\frac{k(k+1)}{\Omega_{1}}}\right)^{2 i_{1}+\mu-1} \frac{1}{i_{1} ! \Gamma\left(i_{1}+\mu\right)} x_{i}^{2 i_{i}+\mu-1}, i=1,2
$$

Na sličan način se dobijaju i gustine verovatnoće signala na ulazima u drugi i treći mikrodiverziti SC prijemnik, respektivno:

$$
\begin{aligned}
& p_{y_{i}}\left(y_{i}\right)=\frac{2 \mu(k+1)^{\frac{\mu+1}{2}}}{k^{\frac{\mu-1}{2}} e^{\mu k} \Omega_{2}^{\mu+1}} y_{i}^{\mu} e^{-\frac{\mu(k+1) y_{i}^{2}}{\Omega_{2}}} \sum_{i_{2}=0}^{\infty}\left(\mu \sqrt{\frac{k(k+1)}{\Omega_{2}}}\right)^{2 i_{2}+\mu-1} \frac{1}{i_{2} ! \Gamma\left(i_{2}+\mu\right)} y_{i}^{2 i_{2}+\mu-1}, i=1,2 \\
& p_{z_{i}}\left(z_{i}\right)=\frac{2 \mu(k+1)^{\frac{\mu+1}{2}}}{k^{\frac{\mu-1}{2}} e^{\mu k} \Omega_{3}^{\mu+1}} z_{i}^{\mu} e^{-\frac{\mu(k+1) z_{i}^{2}}{\Omega_{3}}} \sum_{i_{3}=0}^{\infty}\left(\mu \sqrt{\frac{k(k+1)}{\Omega_{3}}}\right)^{2 i_{3}+\mu-1} \frac{1}{i_{3} ! \Gamma\left(i_{3}+\mu\right)} z_{i}^{2 i_{3}+\mu-1}, i=1,2
\end{aligned}
$$

Kumulativna verovatnoća od $x_{i}, i=1,2$ je

$$
\begin{gathered}
F_{x_{i}}\left(x_{i}\right)=\int_{0}^{x_{i}} d t p_{x_{i}}(t)= \\
=\frac{2 \mu(k+1)^{\frac{\mu+1}{2}}}{k^{\frac{\mu-1}{2}} e^{\mu k} \Omega_{1}^{\mu+1}} \sum_{i_{1}=0}^{\infty}\left(\mu \sqrt{\frac{k(k+1)}{\Omega_{1}}}\right)^{2 i_{1}+\mu-1} \frac{1}{i_{1} ! \Gamma\left(i_{1}+\mu\right)} \int_{0}^{x_{i}} d t \cdot t^{2 i_{1}+2 \mu-1} e^{-\frac{\mu(k+1) t^{2}}{\Omega_{1}}}, i=1,2
\end{gathered}
$$


Nakon rešavanja integrala primenom (Gradshteyn, 2000) dobija se izraz za kumulativnu verovatnoću signala na ulazu u prvi mikrodiverziti SC kombiner:

$$
\begin{gathered}
F_{x_{i}}\left(x_{i}\right)=\frac{\mu(k+1)^{\frac{\mu+1}{2}}}{k^{\frac{\mu-1}{2}} e^{\mu k} \Omega_{1}^{\mu+1}} \sum_{i_{1}=0}^{\infty}\left(\mu \sqrt{\frac{k(k+1)}{\Omega_{1}}}\right)^{2 i_{1}+\mu-1} \frac{1}{i_{1} ! \Gamma\left(i_{1}+\mu\right)} \times \\
\times\left(\frac{\Omega_{1}}{\mu(k+1)}\right)^{i_{1}+\mu} \gamma\left(i_{1}+\mu, \frac{\mu(k+1)}{\Omega_{1}} x_{i}^{2}\right), \quad i=1,2
\end{gathered}
$$

gde $\gamma(\cdot)$ predstavlja donju nepotpunu Gama funkciju. Primenom postupka za dobijanje kumulativne verovatnoće signala na ulazu u prvi mikrodiverziti SC prijemnik mogu se dobiti i kumulativne verovatnoće signala $y_{i} \mathrm{i} z_{i}$ na ulazima u drugi i treći mikrodiverziti SC prijemnik.

Gustina verovatnoće signala na izlazu iz prvog mikrodiverziti SC kombinera je

$$
p_{x}(x)=p_{x_{1}}(x) F_{x_{2}}(x)+p_{x_{2}}(x) F_{x_{1}}(x)=2 p_{x_{1}}(x) F_{x_{2}}(x)
$$

gde je $p_{x 1}$ dato sa (2) a $F_{x 2}$ sa (6). Nakon zamena (2) i (6) u (7) dobija se

$$
\begin{gathered}
p_{x}(x)=4\left(\frac{\mu(k+1)^{\frac{\mu+1}{2}}}{k^{\frac{\mu-1}{2}} e^{\mu k} \Omega_{1}^{\mu+1}}\right)^{2} e^{-\frac{\mu(k+1) x^{2}}{\Omega_{1}}} \sum_{j_{1}=0}^{\infty}\left(\mu \sqrt{\frac{k(k+1)}{\Omega_{1}}}\right)^{2 j_{1}+\mu-1} \frac{1}{j_{1} ! \Gamma\left(j_{1}+\mu\right)} x^{2 j_{1}+2 \mu-1} \times \\
\times \sum_{i_{1}=0}^{\infty}\left(\mu \sqrt{\frac{k(k+1)}{\Omega_{1}}}\right)^{2_{1}+\mu-1} \frac{1}{i_{1} ! \Gamma\left(i_{1}+\mu\right)}\left(\frac{\Omega_{1}}{\mu(k+1)}\right)^{i_{1}+\mu} \gamma\left(i_{1}+\mu, \frac{\mu(k+1)}{\Omega_{1}} x^{2}\right)
\end{gathered}
$$

gde $\gamma(\cdot)$ predstavlja donju nepotpunu Gama funkciju.

Gustina verovatnoće signala na izlazu iz drugog i trećeg mikrodiverziti SC kombinera je

$$
\begin{gathered}
p_{y}(y)=p_{y_{1}}(y) F_{y_{2}}(y)+p_{y_{2}}(y) F_{y_{1}}(y)=2 p_{y_{1}}(y) F_{y_{2}}(y)= \\
=4\left(\frac{\mu(k+1)^{\frac{\mu+1}{2}}}{k^{\frac{\mu-1}{2}} e^{\mu k} \Omega_{2}^{\mu+1}}\right)^{-\frac{\mu(k+1) y^{2}}{\Omega_{2}}} \sum_{j_{2}=0}^{\infty}\left(\mu \sqrt{\frac{k(k+1)}{\Omega_{2}}}\right)^{2 j_{2}+\mu-1} \frac{1}{j_{2} ! \Gamma\left(j_{2}+\mu\right)} y^{2 j_{2}+2 \mu-1} \times \\
\times \sum_{i_{2}=0}^{\infty}\left(\mu \sqrt{\frac{k(k+1)}{\Omega_{2}}}\right)^{2 i_{2}+\mu-1} \frac{1}{i_{2} ! \Gamma\left(i_{2}+\mu\right)}\left(\frac{\Omega_{2}}{\mu(k+1)}\right)^{i_{2}+\mu} \gamma\left(i_{2}+\mu, \frac{\mu(k+1)}{\Omega_{2}} y^{2}\right)
\end{gathered}
$$

i

$$
\begin{gathered}
p_{z}(z)=p_{z_{1}}(z) F_{z_{2}}(z)+p_{z_{2}}(z) F_{z_{1}}(z)=2 p_{z_{1}}(z) F_{z_{2}}(z)= \\
=4\left(\frac{\mu(k+1)^{\frac{\mu+1}{2}}}{k^{\frac{\mu-1}{2}} e^{\mu k} \Omega_{3}^{\mu+1}} e^{-\frac{\mu(k+1) z^{2}}{\Omega_{3}}} \sum_{j_{3}=0}^{\infty}\left(\mu \sqrt{\frac{k(k+1)}{\Omega_{3}}}\right)^{2_{j_{3}+\mu-1}} \frac{1}{j_{3} ! \Gamma\left(j_{3}+\mu\right)} z^{2 j_{3}+2 \mu-1} \times\right. \\
\times \sum_{i_{3}=0}^{\infty}\left(\mu \sqrt{\frac{k(k+1)}{\Omega_{3}}}\right)^{2 i_{3}+\mu-1} \frac{1}{i_{3} ! \Gamma\left(i_{3}+\mu\right)}\left(\frac{\Omega_{3}}{\mu(k+1)}\right)^{i_{3}+\mu} \gamma\left(i_{2}+\mu, \frac{\mu(k+1)}{\Omega_{3}} z^{2}\right)
\end{gathered}
$$

Gustina verovatnoće signala na izlazu iz makrodiverziti SC prijemnika jednaka je gustini verovatnoće signala na izlazu iz onog mikrodiverziti SC prijemnika čija je snaga na ulazu veća od snage signala na ulazu druga dva mikrodiverziti SC prijemnika (Gradshteyn, 2000). Na osnovu ovoga je gustina verovatnoće signala na izlazu iz makrodiverziti SC prijemnika jednaka

$$
\begin{gathered}
p(w)=\int_{0}^{\infty} d \Omega_{1} \int_{0}^{\Omega_{1}} d \Omega_{2} \int_{0}^{\Omega_{1}} d \Omega_{3} p_{x}\left(w / \Omega_{1}\right) p_{\Omega_{1} \Omega_{2} \Omega_{3}}\left(\Omega_{1} \Omega_{2} \Omega_{3}\right)+ \\
+\int_{0}^{\infty} d \Omega_{2} \int_{0}^{\Omega_{2}} d \Omega_{1} \int_{0}^{\Omega_{2}} d \Omega_{3} p_{x}\left(w / \Omega_{2}\right) p_{\Omega_{3} \Omega_{2} \Omega_{3}}\left(\Omega_{1} \Omega_{2} \Omega_{3}\right)+ \\
+\int_{0}^{\infty} d \Omega_{3} \int_{0}^{\Omega_{3}} d \Omega_{1} \int_{0}^{\Omega_{3}} d \Omega_{2} p_{x}\left(w / \Omega_{3}\right) p_{\Omega_{1} \Omega_{2} \Omega_{3}}\left(\Omega_{1} \Omega_{2} \Omega_{3}\right)= \\
=I_{1}+I_{2}+I_{3}
\end{gathered}
$$

gde je $p_{x}\left(w / \Omega_{1}\right), p_{x}\left(w / \Omega_{2}\right)$ i $p_{x}\left(w / \Omega_{3}\right)$ dato sa (8), (9) i (10), respektivno. Združena gustina verovatnoće snaga $\Omega_{1}, \Omega_{2}$ i $\Omega_{3}$ je data sa:

$$
\begin{gathered}
p_{\Omega_{1} \Omega_{2} \Omega_{3}}\left(\Omega_{1} \Omega_{2} \Omega_{3}\right)=\frac{1}{\Gamma(c)\left(1-\rho^{2}\right) \rho^{c-1} \Omega_{0}^{c+2}} \sum_{i_{1}=0}^{\infty}\left(\frac{\sqrt{\rho}}{\Omega_{0}(1-\rho)}\right)^{i_{1}+c-1} \frac{1}{i_{1} ! \Gamma\left(i_{1}+c\right)} \times \\
\times \sum_{i_{2}=0}^{\infty}\left(\frac{\sqrt{\rho}}{\Omega_{0}(1-\rho)}\right)^{2 i_{2}+c-1} \frac{1}{i_{2} ! \Gamma\left(i_{2}+c\right)} \Omega_{1}^{i_{1}+c-1} \Omega_{2}^{i_{1}+i_{2}+c-1} \Omega_{3}^{i_{1}+c-1} e^{-\frac{\Omega_{1}+\Omega_{2}(1+\rho)+\Omega_{3}}{\Omega_{0}(1-\rho)}}
\end{gathered}
$$


Integral $I_{1}$ je jednak

$$
\begin{gathered}
I_{1}=\int_{0}^{\infty} d \Omega_{1} \int_{0}^{\Omega_{1}} d \Omega_{2} \int_{0}^{\Omega_{1}} d \Omega_{3} p_{x}\left(w / \Omega_{1}\right) p_{\Omega_{1} \Omega_{2} \Omega_{3}}\left(\Omega_{1} \Omega_{2} \Omega_{3}\right)= \\
=\frac{4 \mu^{2}(k+1)^{\mu+1}}{k^{\mu-1} e^{2 \mu k}} \sum_{i_{2}=0}^{\infty}(\mu \sqrt{k(k+1)})^{2 i_{2}+\mu-1} w^{2 i_{2}+2 \mu-1} \frac{1}{i_{2} ! \Gamma\left(i_{1}+\mu\right)} \times \sum_{i_{1}=0}^{\infty}(\mu \sqrt{k(k+1)})^{2 i_{1}+\mu-1} \\
\times \frac{1}{i_{1} ! \Gamma\left(i_{1}+\mu\right)} \frac{1}{(\mu(k+1))^{i_{1}+\mu}} \frac{1}{\Gamma(c)\left(1-\rho^{2}\right) \rho^{c-1} \Omega_{0}^{c+2}} \sum_{i_{3}=0}^{\infty}\left(\frac{\sqrt{\rho}}{\Omega_{0}(1-\rho)}\right)^{2 i_{3}+c-1} \times \\
\times \frac{1}{i_{3} ! \Gamma\left(i_{3}+c\right)} \sum_{i_{4}=0}^{\infty}\left(\frac{\sqrt{\rho}}{\Omega_{0}(1-\rho)}\right)^{2 i_{4}+c-1} \frac{1}{i_{4} ! \Gamma\left(i_{4}+c\right)} \times \\
\times \int_{0}^{\infty} d \Omega_{1} \Omega_{1}^{i_{3}+c-1-i_{1}-\frac{\mu-1}{2}-i_{2}-\frac{\mu-1}{2}-2(\mu+1)} e^{-\frac{\mu(k+1) w^{2}}{\Omega_{1}}-\frac{\Omega_{1}}{\Omega_{0}(1-\rho)} \gamma\left(i_{1}+\mu, \frac{\mu(k+1)}{\Omega_{1}} w^{2}\right) \times} \\
\quad \times \int_{0}^{\Omega_{1}} d \Omega_{2} \Omega_{2}^{i_{3}+i_{4}+c-1} e^{-\frac{\Omega_{2}(1+\rho)}{\Omega_{0}(1-\rho)} \Omega_{1}} d \Omega_{0} \Omega_{3}^{i_{4}+c-1} e^{-\frac{\Omega_{3}}{\Omega_{0}(1-\rho)}}
\end{gathered}
$$

Nakon primene (Gradshteyn, 2000) za rešavanje drugog i trećeg integrala u (13), $I_{1}$ postaje

$$
\begin{gathered}
I_{1}=\frac{4 \mu^{2}(k+1)^{\mu+1}}{k^{\mu-1} e^{2 \mu k}} \sum_{i_{2}=0}^{\infty}(\mu \sqrt{k(k+1)})^{2 i_{2}+\mu-1} w^{2 i_{2}+2 \mu-1} \frac{1}{i_{2} ! \Gamma\left(i_{1}+\mu\right)} \sum_{i_{1}=0}^{\infty}(\mu \sqrt{k(k+1)})^{2 i_{1}+\mu-1} \times \\
\times \frac{1}{i_{1} ! \Gamma\left(i_{1}+\mu\right)} \frac{1}{(\mu(k+1))^{i_{1}+\mu}} \frac{1}{\Gamma(c)\left(1-\rho^{2}\right) \rho^{c-1} \Omega_{0}^{c+2}} \sum_{i_{3}=0}^{\infty}\left(\frac{\sqrt{\rho}}{\Omega_{0}(1-\rho)}\right)^{2 i_{3}+c-1} \times \\
\times \frac{1}{i_{3} ! \Gamma\left(i_{3}+c\right)} \sum_{i_{4}=0}^{\infty}\left(\frac{\sqrt{\rho}}{\Omega_{0}(1-\rho)}\right)^{2 i_{4}+c-1} \frac{1}{i_{4} ! \Gamma\left(i_{4}+c\right)}\left(\Omega_{0}(1-\rho)\right)^{i_{4}+c} \times \\
\times \int_{0}^{\infty} d \Omega_{1} \Omega_{1}^{i_{3}+c-i_{1}-i_{2}-3 \mu-2} e^{-\frac{\mu(k+1) w^{2}}{\Omega_{1}}}-\frac{\Omega_{1}}{\Omega_{0}(1-\rho)} \gamma\left(i_{1}+\mu, \frac{\mu(k+1)}{\Omega_{1}} w^{2}\right) \times \\
\times \gamma\left(i_{3}+i_{4}+c, \frac{(1+\rho) \Omega_{1}}{\Omega_{0}(1-\rho)}\right) \gamma\left(i_{4}+c, \frac{\Omega_{1}}{\Omega_{0}(1-\rho)}\right)
\end{gathered}
$$

Nakon razvoja Gama funkcije

$$
\gamma(n, x)=\frac{1}{n} x^{n} e^{-x}{ }_{1} F_{1}(1, n+1, x)=\frac{1}{n} x^{n} e^{-x} \sum_{i=0}^{\infty} \frac{n !}{(n+i) !} x^{i}
$$

i primenom (Gradshteyn, 2000) dobija se

$$
\begin{aligned}
I_{1}=\frac{4 \mu^{2}(k+1)^{\mu+1}}{k^{\mu-1} e^{2 \mu k}} \sum_{i_{2}=0}^{\infty}(\mu \sqrt{k(k+1)})^{2 i_{2}+\mu-1} w^{2 i_{2}+2 \mu-1} \frac{1}{i_{2} ! \Gamma\left(i_{1}+\mu\right)} \sum_{i_{1}=0}^{\infty}(\mu \sqrt{k(k+1)})^{2 i_{1}+\mu-1} \times \\
\times \frac{1}{i_{1} ! \Gamma\left(i_{1}+\mu\right)} \frac{1}{(\mu(k+1))^{i_{1}+\mu}} \frac{1}{\Gamma(c)\left(1-\rho^{2}\right) \rho^{c-1} \Omega_{0}^{c+2}} \sum_{i_{3}=0}^{\infty}\left(\frac{\sqrt{\rho}}{\Omega_{0}(1-\rho)}\right)^{2 i_{3}+c-1} \times \\
\quad \times \frac{1}{i_{3} ! \Gamma\left(i_{3}+c\right)} \sum_{i_{4}=0}^{\infty}\left(\frac{\sqrt{\rho}}{\Omega_{0}(1-\rho)}\right)^{2 i_{4}+c-1} \frac{1}{i_{4} ! \Gamma\left(i_{4}+c\right)}\left(\Omega_{0}(1-\rho)\right)^{i_{4}+c} \times \\
\quad \times \frac{1}{i_{1}+c}\left(\mu(k+1) w^{2}\right)^{i_{1}+c} \sum_{j_{1}=0}^{\infty} \frac{\left(i_{1}+c\right) !}{\left(i_{1}+c+j_{1}\right) !}\left(\mu(k+1) w^{2}\right)^{j_{1}} \times \\
\times \frac{1}{i_{3}+i_{4}+c} \frac{1}{\left(\Omega_{0}(1-\rho)\right)^{i_{3}+i_{4}+c}} \sum_{j_{2}=0}^{\infty} \frac{\left(i_{3}+i_{4}+c\right) !}{\left(i_{3}+i_{4}+c+j_{2}\right) !} \frac{1}{\left(\Omega_{0}(1-\rho)\right)^{j_{2}}} \times \\
\quad \times \frac{1}{i_{4}+c} \frac{1}{\left(\Omega_{0}(1-\rho)\right)^{i_{4}+c}} \sum_{j_{3}=0}^{\infty} \frac{\left(i_{4}+c\right) !}{\left(i_{4}+c+j_{3}\right) !\left(\Omega_{0}(1-\rho)\right)^{j_{3}}} \times \\
\quad \times\left(\frac{2 \mu(k+1) w^{2} \Omega_{0}(1-\rho)}{2+\rho}\right) \\
\quad \times K_{-2 i_{1}-i_{2}+2 i_{3}+2 i_{4}-j_{1}+j_{2}+j_{3}-3 \mu+2 c-2}\left(2 \sqrt{\frac{2 \mu\left(i_{1}-i_{2}+2 i_{3}+2 i_{4}-j_{1}+j_{2}+j_{3}-3 \mu+2 c-2\right.}{2}} \times\right. \\
\Omega_{0}(1-\rho)
\end{aligned}
$$

gde je $K_{n}(x)$ modifikovana Beselova funkcija druge vrste, $n$-tog reda i argumenta $x$. 
Primenom metode za računanje integrala $I_{1}$, rešavaju se i integral $I_{2}$ i $I_{3}$ :

$$
I_{2}=\int_{0}^{\infty} d \Omega_{2} \int_{0}^{\Omega_{2}} d \Omega_{1} \int_{0}^{\Omega_{2}} d \Omega_{3} p_{y}\left(w / \Omega_{2}\right) p_{\Omega_{1} \Omega_{2} \Omega_{3}}\left(\Omega_{1} \Omega_{2} \Omega_{3}\right)
$$

i

$I_{3}=\int_{0}^{\infty} d \Omega_{3} \int_{0}^{\Omega_{3}} d \Omega_{1} \int_{0}^{\Omega_{3}} d \Omega_{2} p_{z}\left(w / \Omega_{3}\right) p_{\Omega_{1} \Omega_{2} \Omega_{3}}\left(\Omega_{1} \Omega_{2} \Omega_{3}\right)$.

\section{NUMERIČKI REZULTATI}

Na Sl. 2 je prikazana gustina verovatnoće signala $w$ na izlazu iz makrodiverziti sistema za različite vrednosti dubine osenčenosti kanala $c$ i Rajsovog $k$ faktora. Broj klastera $\mu=1.5$. Vrh gustine verovatnoće je viši za visoke vrednosti dubine osenčenosti kanala $c$ i Rajsovog $k$ faktora.

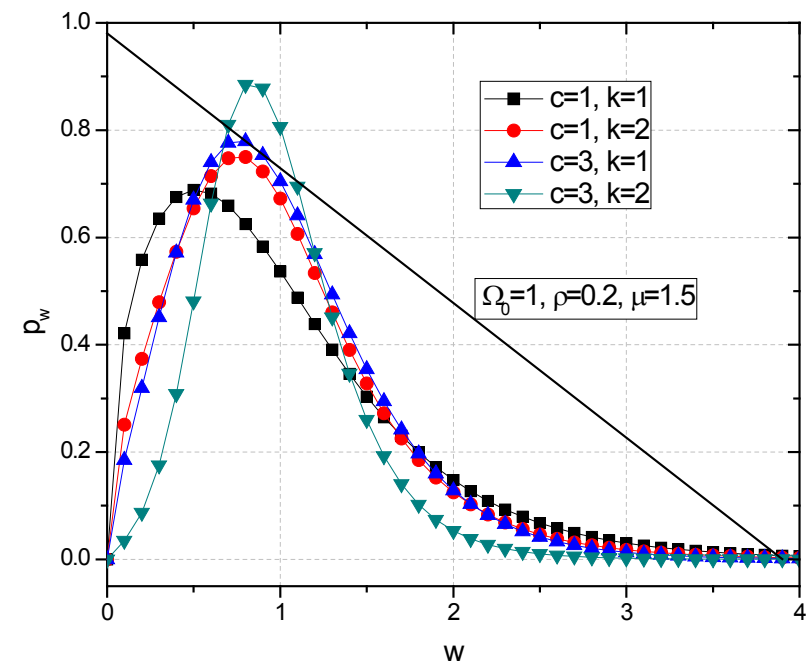

Slika 2. Gustina verovatnoće signala $w$ na izlazu iz makrodiverziti sistema za različite vrednosti dubine osenčenosti kanala $c$ i Rajsovog $k$ faktora.
$\mathrm{Na}$ Sl. 3 je data gustina verovatnoće signala $w$ na izlazu iz makrodiverziti sistema za različite vrednosti broja klastera $\mu$ i dubine osenčenosti kanala $c$. Rajsov faktor iznosi $k=1$. Viši vrhovi gustine verovatnoće se dobijaju za više vrednosti broja klastera $\mu$ i za više vrednosti dubine osenčenosti kanala $c$.

$\mathrm{Na}$ Sl. 4 je prikazana promena Gustina verovatnoće signala $w$ na izlazu iz makrodiverziti sistema za različite vrednosti Rajsovog $k$ faktora i broja klastera $\mu$. Dubina osenčenosti kanala je $c=1$. Sa povećanjem Rajsovog $k$ faktora i broja klastera vrhovi gustine verovatnoće su više izraženiji. Koficijent korelacije za sva tri grafika je $\rho=0.2$, srednja kvadratna promena snage $\Omega_{0}=1$.

\section{REZIME}

U ovom radu razmatran je diverziti sistem sa tri mikrodiverziti SC prijemnika i jednim makrodiverziti SC prijemnikom. $\mathrm{Na}$ ulazima u mikrodiverziti SC prijemnike prisutan je nezavisni $k-\mu$ feding i korelisani spori Gama feding. Mikrodiverziti

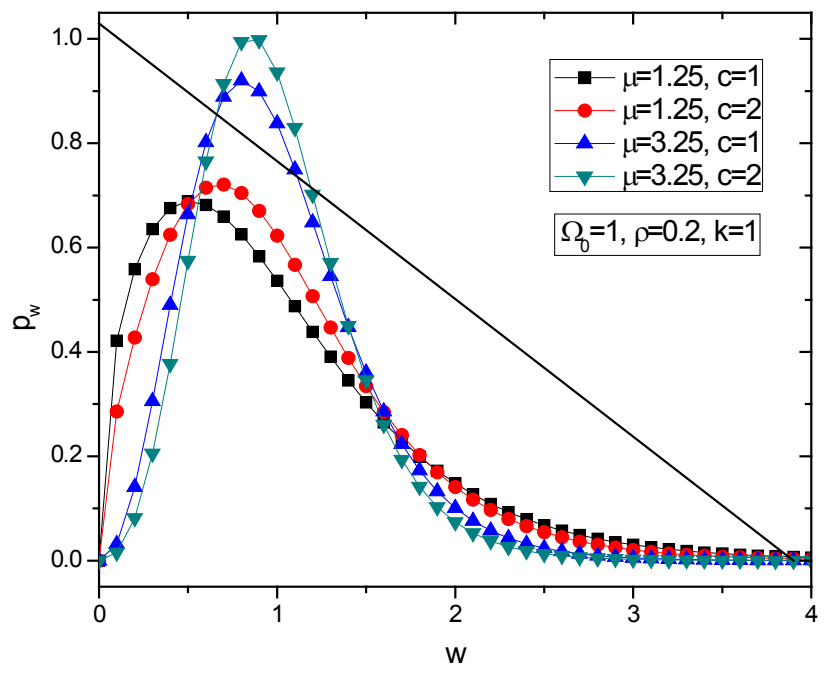

Slika 3. Gustina verovatnoće signala $w$ na izlazu iz makrodiverziti sistema za različite vrednosti broja klastera $\mu$ i dubine osenčenosti kanala $c$.

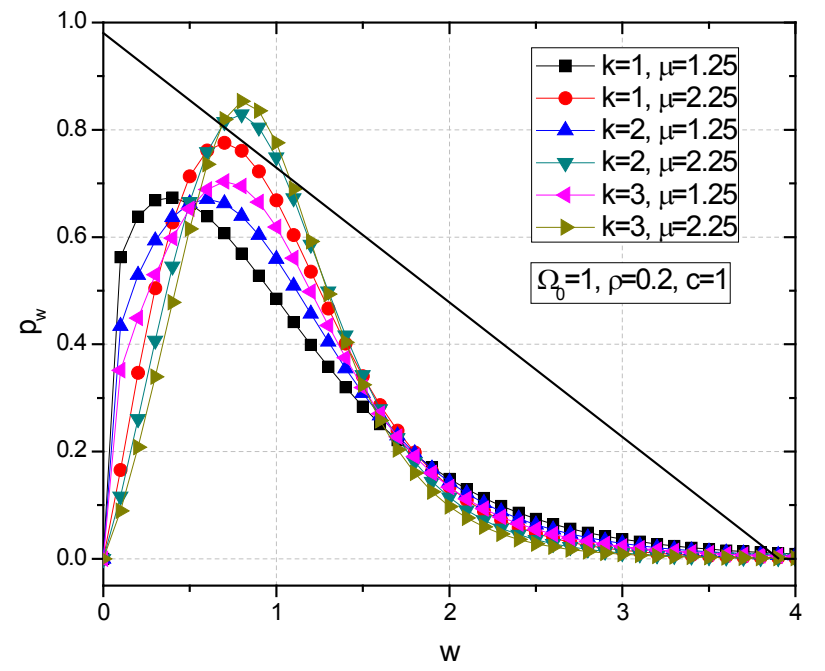

Slika 4. Gustina verovatnoće signala $w$ na izlazu iz makrodiverziti sistema za različite vrednosti Rajsovog $k$ faktora i broja klastera $\mu$. 
SC prijemnik smanjuje uticaj brzog fedinga na performanse sistema, a makrodiverziti SC prijemnik smanjuje uticaj sporog fedinga na performanse sistema. Za ovaj sistem izračunati su gustina verovatnoća signala na izlazu iz diverziti sistema. Gustina verovatnoća signala je važne statistička karakteristika na osnovu koje se računaju druge statističke karakteristike prvog i drugog reda.

Kada je parametar $k=0$, onda $k-\mu$ raspodela prelazi u Nakagami- $m$ raspodelu, a kada je $\mu=1$, Rajsova raspodela se može dobiti iz $k$ - $\mu$ raspodele. Kada je $k=0$ i $\mu=1, k$ - $\mu$ raspodela prelazi $\mathrm{u}$ Rejlijevu raspodelu. Kada parametar $\mu$ opada, oštrina uticaja fedinga raste, a kada parametar $\mu$ raste, oštrina uticaja fedinga opada. Kada oštrina uticaja fedinga raste performanse sistema pogoršavaju. Oštriji uticaj fedinga se javlja kada je Rajsov $k$ faktor manji.

\section{LITERATURA}

Gradshteyn, I.S., \& Ryzhik, I.M. (2000). Table of Integrals, Series and Products. San Diego: Academic Press.

Panic, S., Stefanovi, M., Anastasov, J. \& Spalevic, P. (2013). Fading and Interference Mitigation in Wireless Communications. USA: CRC Press.

Panic, S., Stefanovic, D., Petrovic, I., Stefanovic, M., Anastasov, J., \& Krstic, D. (2011). Second-order statistics of selection macro-diversity system operating over Gamma shadowed $\kappa-\mu$ fading channels. EURASIP Journal on Wireless Communications and Networking. 2011 (151), 1-7. DOI: 10.1186/16871499-2011-151
Proakis, J. (2001). Digital Communications, 4nd ed. New York: McGraw-Hill.

Sekulovic, N., \& Stefanović, M. (2012). Performance Analysis of System with Micro- and Macrodiversity Reception in Correlated Gamma Shadowed Rician Fading Channels. Wireless Personal Communications, 65(1), 143-156. DOI: 10.1007/s11277-011-0232-8

Shankar, P. M. (2008). Analysis of microdiversity and dual channel macrodiversity in shadowed fading channels using a compound fading model. International Journal of Electronics and Communications (AEUE), 62(6), 445-449. DOI: 10.1016/j.aeue.2007.06.008

Simon, M.K., \& Alouini, M.S. (2000). Digital Communication over Fading Channels. USA: John Wiley \& Sons.

Stuber, G.L. (2003). Mobile communication, 2nd ed. Dordrecht: Kluwer Academic Publisher.

Yacoub, M.D. (2007). The $\eta-\mu$ distribution and the $\kappa-\mu$ distribution. IEEE Antennas and Propagation Magazine, 49(1), 68-81. DOI: 10.1109/MAP.2007.370983 